\title{
Evaluation of the accuracy and angular resolution of q-ball imaging
}

\author{
Kuan-Hung Cho, ${ }^{\mathrm{a}}$ Chun-Hung Yeh, ${ }^{\mathrm{b}}$ Jacques-Donald Tournier, \\ Yi-Ping Chao, ${ }^{\mathrm{a}}$ Jyh-Horng Chen, ${ }^{\mathrm{a}, *}$ and Ching-Po Lin ${ }^{\mathrm{b}, \mathrm{d}, \mathrm{e}, *}$ \\ anterdisciplinary MRI/MRS Lab, Department of Electrical Engineering, National Taiwan University, Taipei, Taiwan \\ ${ }^{\mathrm{b}}$ Department of Biomedical Imaging and Radiological Sciences, National Yang-Ming University, Taipei, Taiwan \\ ${ }^{\mathrm{c}}$ Brain Research Institute, Melbourne, Australia \\ ${ }^{\mathrm{d}}$ Lab for Brain Connectivity, Institute of Neuroscience, National Yang-Ming University, Taipei, Taiwan \\ ${ }^{\mathrm{e}}$ MRI Research Center, National Yang-Ming University, Taipei, Taiwan
}

Received 24 December 2007; revised 9 March 2008; accepted 26 March 2008

Available online 9 April 2008

Q-ball imaging (QBI) has been proposed for the mapping of multiple intravoxel fiber structures using the Funk-Radon transform on high angular resolution diffusion images (HARDI). However, the accuracy and the angular resolution of QBI to define fiber orientations and its dependence on diffusion imaging parameters remain unclear. The phantom models, made up of sheets of parallel capillaries filled with water, were designed to evaluate the accuracy and the angular resolution of QBI at different $|q|$ values. With an inner diameter of $20 \mu \mathrm{m}$ and an outer diameter of $90 \mu \mathrm{m}$, the capillaries afforded a restrictive environment compared with the diffusion measurement scale. Further, the angular resolutions of $\mathrm{QBI}$ at various $|q|$ value were also quantified on the corpus callosum in the human brain. The full width at half maximum (FWHM) of the main lobe of normalized orientation distribution function (nODF) was calculated and adopted to quantify the angular resolution of QBI. With the phantom model, a higher $|q|$ value resulted in worse accuracy but better angular resolution for QBI. The same trend where a higher $|q|$ value yielded a better angular resolution was also observed in the human study. Upon comparison of QBI with T2WI, QBI with $|q|=277 \mathrm{~cm}^{-1}$ $\left(b=3000 \mathrm{~s} / \mathrm{mm}^{2}\right)$ was found to be insufficient to differentiate capillaries crossing at $45^{\circ}$. However, when encoding with $|q|=320,358$, and $392 \mathrm{~cm}^{-1}\left(b=4000,5000\right.$, and $\left.6000 \mathrm{~s} / \mathrm{mm}^{2}\right)$, the deviation angles between the primary $\mathrm{ODF}$ and the $45^{\circ}$ phantoms were $-4.91^{\circ} \pm 2.72^{\circ}$, $-1.37^{\circ} \pm 2.32^{\circ}$, and $-0.69^{\circ} \pm 1.54^{\circ}$ with adequate signal-to-noise ratio (SNR). These results were consistent with the FWHM-nODF, which showed that a $|q|$ value of $320 \mathrm{~cm}^{-1}$ was the threshold to resolve capillaries intersecting at $45^{\circ}$. Additionally, it was demonstrated in both the phantom model and the human brain that QBI encoding with lower $|q|$ values may result in underestimation of the orientations of the

\footnotetext{
* Corresponding authors. J.-H. Chen is to be contacted at Department of Electrical Engineering, National Taiwan University, No. 1, Sec. 4, Roosevelt Road, Taipei, Taiwan. Fax: +886 2 23648238. C.-P. Lin, Institute of Neuroscience, National Yang-Ming University, 155 Li-Nong Street, Sec. 2, Taipei, 112, Taiwan. Fax: +886228262285.

E-mail addresses: jyhhchen@gmail.com (J.-H. Chen), cplin@ym.edu.tw (C.-P. Lin).

Available online on ScienceDirect (www.sciencedirect.com).
}

crossing fibers. In conclusion, QBI was found to accurately resolve crossing fiber orientations and was highly dependent on the selected $|q|$ value.

(C) 2008 Elsevier Inc. All rights reserved.

Keywords: Q-ball; Phantom model; Optimization

\section{Introduction}

Diffusion Magnetic Resonance Imaging (MRI) can be utilized to map tissue microstructure by probing direction-dependent water molecule diffusion within the biological architecture (Callaghan, 1993; Grannell and Mansfield, 1975). During the last decade, various models have been developed in order to map the displacement of water molecules and have provided significant information regarding the microstructure dimensions, water mobility in specific anatomical compartments, and the orientation of tissue macrostructures (Assaf and Basser, 2005; Assaf and Cohen, 1999; King et al., 1994). Diffusion tensor imaging (DTI) was the first model to map the intravoxel architecture by measuring the water self-diffusion tensor (Basser et al., 1994). The orientation determined by the principal eigenvector of the diffusion tensor was inferred to be parallel to the mean axonal tract orientation within each magnetic resonance (MR) voxel and has been widely applied to map the neuronal tracts in vivo (Basser et al., 2000; Behrens et al., 2003; Conturo et al., 1999; Lin et al., 2001; Mori et al., 1999).

The diffusion tensor, which possesses only one peak orientation, brings about an ambiguous principal orientation in voxels containing multi-fiber structures (Alexander et al., 2001; Wiegell et al., 2000). This drawback is noticeable because multiple structures of human cerebral white matter occur within the millimeter width of a typical MRI voxel. This dilemma led to the further development of methods dedicated to resolving the microscopic diffusion function 
using high angular resolution diffusion imaging (HARDI) (Alexander et al., 2002; Anderson, 2005; Descoteaux et al., 2006; Frank, 2001, 2002; Ozarslan and Mareci, 2003; Tournier et al., 2004; Tuch et al., 2002; Zhan et al., 2003).

Q-ball imaging (QBI), one of the HARDI methods, directly derives the diffusion orientation distribution function (ODF) from diffusion images using the Funk-Radon transform (FRT). It is feasible to resolve the crossing of white matter fibers within a voxel as well as the insertion of white matter into the cortex (Tuch, 2004; Tuch et al., 2003). QBI has also been used to identify the structures of the human brain using 3D tractography (Campbell et al., 2005; Perrin et al., 2005a). Perrin et al. showed the capability of QBI to resolve rayon fibers crossing at $90^{\circ}$ on a clinical MRI (Perrin et al., 2005b). However, the long echo time resulted in a low SNR and therefore diluted the accuracy of QBI. To the best of our knowledge, the accuracy of QBI to define the fiber orientation and the ability of QBI to define the angle of separation (i.e., angular resolution) have not been quantified with respect to $|q|$ values.

The capability of QBI to differentiate crossing fibers depends on the $q$ sphere encoding points and the wave vector $q$, defined as $\gamma \delta g$ ( $\gamma$ : gyromagnetic ratio, $\delta$ : time duration of the diffusionencoding gradient, and $g$ : diffusion-encoding gradient vector). The angular resolution can be improved by increasing the sampling points on a sphere in $q$ space; however, despite the fact that the angular resolution and the SNR can be enhanced, this is generally limited by feasible MRI scan time. The wave vector $q$ yields a spatial resolution $\Delta r=\alpha_{0} / 2 \pi|q|$ defined by the width of the Bessel beam, where $\alpha_{0}$ is the first zero of the zeroth-order Bessel function. For large $|q|$, the width of the Bessel beam becomes narrow and consequently enhances the resolution while reciprocally lowering the SNR (Tuch, 2004). Even though a reciprocal relationship between the spatial resolution and the SNR has been pointed out, most MR users are still unaware of the imaging parameters necessary to achieve better results when using QBI analysis.

The goals of this study were to quantify the accuracy of QBI to define the fiber orientation in relation to the $|q|$ value and the imaging SNR and to assess the angular resolution of QBI to resolve intravoxel fiber crossings at various $|q|$ values for both phantom and human studies. The angular resolution was defined as the FWHM (full width at half maximum) of the main lobe of the normalized ODF (nODF). Based on the assumption that the diffusion-weighted signal $\mathrm{E}(q)$ was an axially symmetric function, a simplified encoding scheme was proposed in order to shorten the acquisition time for measuring the FWHM of the main lobe of the nODF (FWHM-nODF). The capillary phantoms were designed to quantify the accuracy of QBI in resolving the fiber orientation and to assess the feasibility of estimating the angular resolution of QBI by measuring the FWHM-nODF. The results showed that the accuracy of QBI was worse with higher $|q|$ values or lower SNRs, and the angular resolution was highly dependent on the $|q|$ value. When the SNR of the reference image $\left(b=0 \mathrm{~s} / \mathrm{mm}^{2}\right)$ was 22 , the optimum $|q|$ value for the capillary phantoms was approximately $392 \mathrm{~cm}^{-1}\left(b=6000 \mathrm{~s} / \mathrm{mm}^{2}\right)$ based on a combination of accuracy and angular resolution. On a healthy human subject, the FWHMnODF showed the same trend where higher $|q|$ values yielded a narrower FWHM-nODF compared to that of the phantom models. The QBI was also acquired on centrum semiovale, which contained three-way fiber crossings. The results showed that some
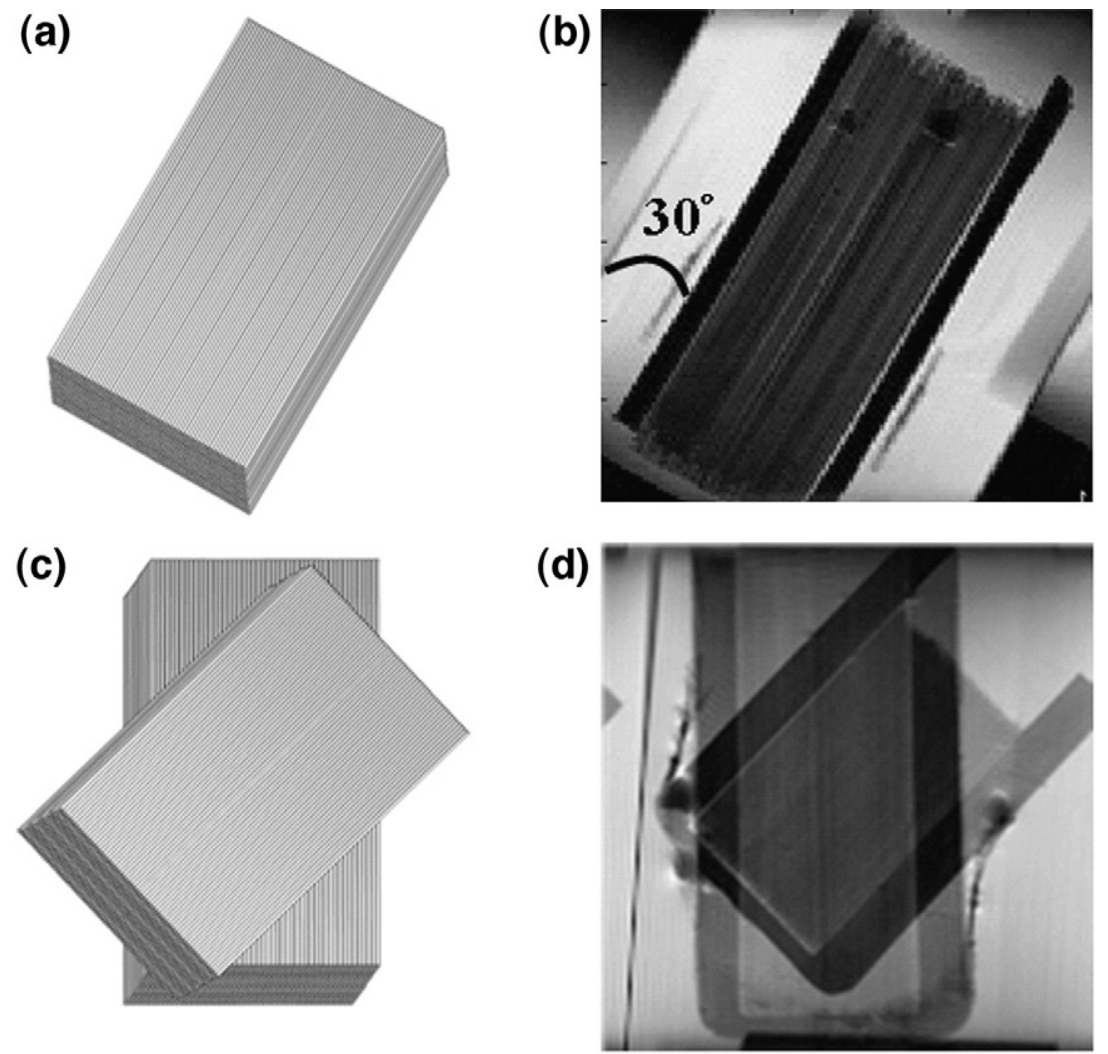

Fig. 1. The design of capillary phantoms. Capillaries were filled with water and sheets were densely stacked to emulate compact fiber bundles (a) and fibers crossing at $45^{\circ}$ (c). High resolution T2WI of the two phantom models served as a reference to quantify the error of QBI (b, d). 
intravoxel fiber crossings were resolved at high $|q|$ values but not at low $|q|$ values because of the better angular resolution at higher $|q|$ values.

\section{Materials and methods}

\section{Phantom models}

Water-filled plastic capillaries (TSP020090, Polymicro Technologies, L.L.C., USA) were used for the design of the phantom model. With an inner diameter (i.d.) of $20 \mu \mathrm{m}$ and an outer diameter (o.d.) of $90 \mu \mathrm{m}$, this capillary phantom provided a restrictive environment with comparable diffusion scale. Two phantom models were designed: a unidirectional phantom and a $45^{\circ}$ intersecting capillaries model. Capillaries were cut to $2 \mathrm{~cm}$ in length and densely packed in order to emulate compact fiber bundles (Fig. 1a). The reference orientation of the unidirectional phantom model was obtained by measuring the separation angle on the T2-weighted image (T2WI) and was approximately $30^{\circ}$ left from the readout direction (Fig. 1b). The intersecting phantom model was designed by stacking up two sheets of capillaries at a separation angle of $45^{\circ}$ (Figs. 1c, d). It was used to explore the capability of QBI to resolve intravoxel fiber crossings in relation to the $|q|$ values and to verify the reliability of the quantification of the angular resolution of QBI using FWHM-nODF.
MR images of the phantom models were acquired using a 9.4 Tesla MRI system (Bruker Companies, Ettlingen, Germany) with a maximum gradient strength of $140 \mathrm{G} \mathrm{cm}^{-1}$. A quadrature volume coil (i.d. $=25 \mathrm{~mm}$ ) was used for RF transmission and reception. The T2WI and diffusion-weighted images (DWIs) were acquired with a field of view (FOV) of $25 \times 25 \mathrm{~mm}$ and a slice thickness of $2 \mathrm{~mm}$. We used a RARE (Rapid Acquisition with Relaxation Enhancement) sequence to obtain the T2WI with $\mathrm{TR} / \mathrm{TE}=2500 / 40.4 \mathrm{~ms}$ and a matrix size of $128^{2}$, yielding an in-plane resolution of $0.20 \mathrm{~mm}$. DWIs were acquired with a stimulated echo-pulsed gradient sequence with $\mathrm{TR} / \mathrm{TE}=3000 / 13.8 \mathrm{~ms}$ and a matrix size of $32^{2}$, yielding an in-plane resolution of $0.78 \mathrm{~mm}$.

\section{Measurement of the FWHM-nODF}

The angular resolution of QBI was quantified by measuring the FWHM-nODF with respect to various $|q|$ values. To simplify the measurement, we posited that uniform fibers within each MR voxel were axially symmetrical (Tournier et al., 2004) and consequently, both the diffusion-weighted signal $\mathrm{E}(q)$ and the ODF $\mathrm{O}(u)$ were axially symmetrical functions. The encoding scheme can be simplified into a single circle in $q$ space with 60 non-collinear diffusionencoding directions, yielding an angular sampling resolution of $6^{\circ}$ (Fig. 2a). Five $|q|$ values, 196, 253, 320, 392, and $452 \mathrm{~cm}^{-1}(b=1.5$, $2.5,4.0,6.0$, and $\left.8.0 \times 10^{3} \mathrm{~s} / \mathrm{mm}^{2}\right)$, were measured. The FWHM- (a)
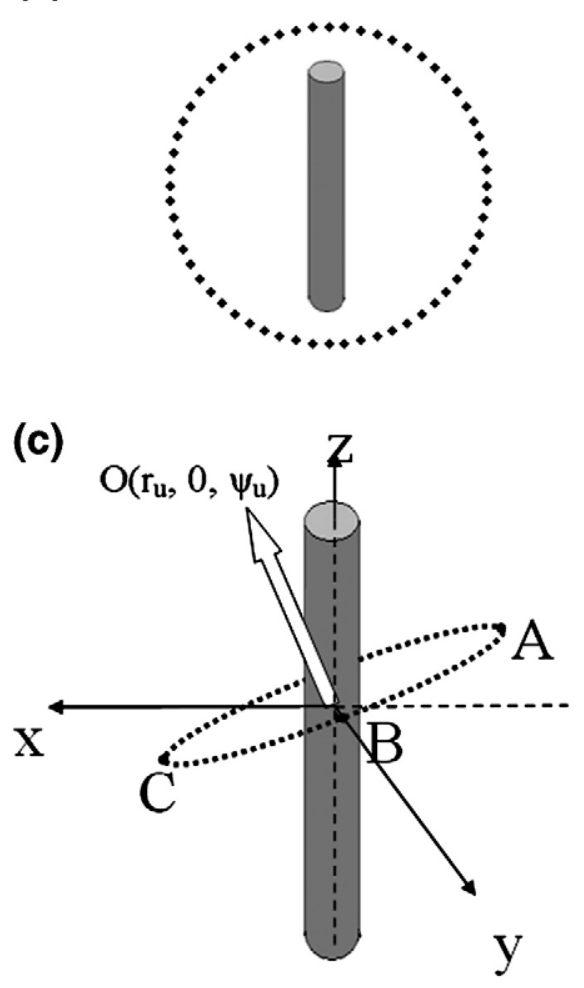

(b)

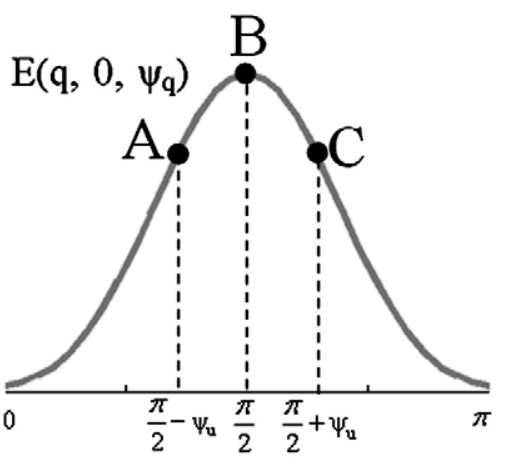

(d)

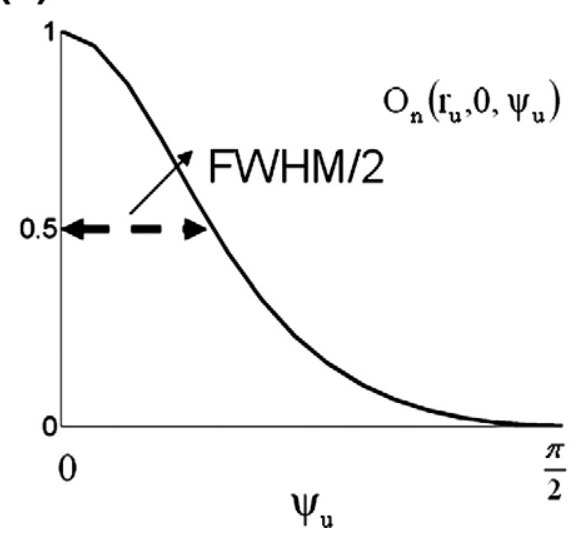

Fig. 2. The illustration for the measurement of the FWHM-nODF using a time-efficient encoding scheme. (a) The fiber was assumed to be on the $z$-axis without losing generality. 60 gradient directions were uniformly distributed on a circle. (b) The distribution of the diffusion signal E( $q$, 0 , $\left.\psi_{q}\right)$, plotted as a function of colatitudes. (c) The illustration of the equator corresponding to a given $\mathrm{ODF} \mathrm{O}\left(r_{u}, 0, \psi_{u}\right)$. The diffusion signals at the equator were extracted from $\mathrm{E}\left(q, 0, \psi_{q}\right)$ and the ODF O $\left(r_{u}, 0, \psi_{u}\right)$ was obtained using Eq. (6). (d) The plot of nODF $\mathrm{O}_{\mathrm{n}}\left(r_{u}, 0, \psi_{u}\right)$ from $\psi_{u}=0$ to $\psi_{u}=\pi$. The FWHM-nODF was defined as twice the width at half maximum because the nODF was theoretically symmetric to $\psi_{u}=0$. 


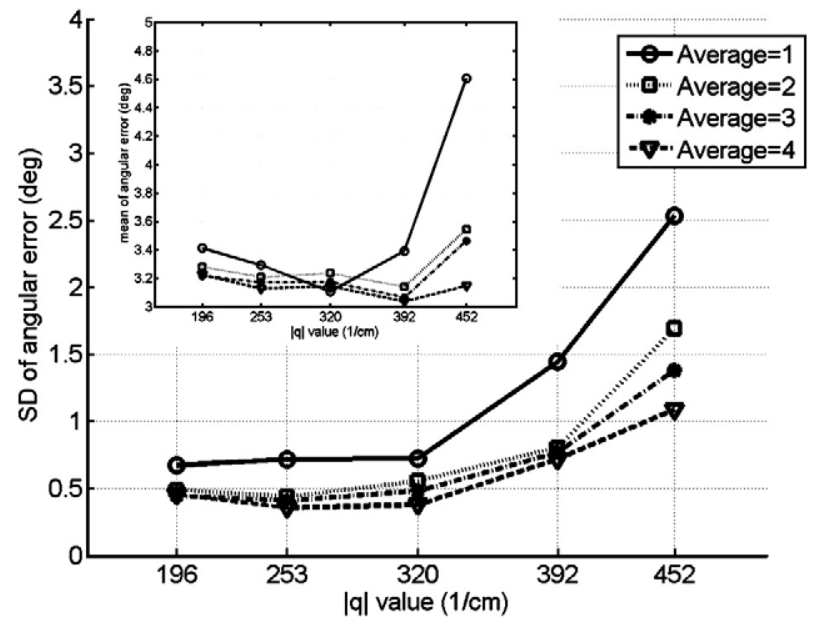

Fig. 3. The mean (subplot) and the SD of the angular error measured from all the voxels, plotted as a function of the $|q|$ values with four different SNRs.

nODF was then derived from the diffusion echo signals $\mathrm{E}(q)$ using Eq. (6) (see Appendix).

\section{Accuracy and angular resolution of $Q B I$}

In order to quantify the accuracy of QBI to define compact fiber bundles in relation to the $|q|$ value, high angular resolution diffusion images were acquired at five $|q|$ values: 196, 253, 320, 392 , and $452 \mathrm{~cm}^{-1}\left(b=1.5,2.5,4.0,6.0\right.$, and $\left.8.0 \times 10^{3} \mathrm{~s} / \mathrm{mm}^{2}\right)$. The diffusion-encoding scheme constituted of 92 diffusion-encoding directions (3-fold tessellated icosahedron), each corresponding to a point in the space of spatial modulation, yielding an angular sampling resolution of $23^{\circ}$. The gradient duration was $3 \mathrm{~ms}$ and the diffusion time was $100 \mathrm{~ms}$. This provided an Einstein length of molecular diffusion of approximately $(2 \mathrm{D} \Delta)^{1 / 2}=20 \mu \mathrm{m}$, equivalent to the inner diameter of the capillaries. Each QBI data set was acquired with four separate repetitions. The SNRs of the reference images $\left(b=0 \mathrm{~s} / \mathrm{mm}^{2}\right)$ were 11.3, 16.0, 19.6, and 22.6 using $1,2,3$, and 4 averages, respectively. After QBI reconstruction, the angular error, defined as the angle between the primary orientation of the ODF and the standard orientation of the phantom model measured on the T2WI, was computed for each voxel.

We quantified the capability of QBI to resolve two intersecting fiber populations and the validity of the FWHM-nODF using the $45^{\circ}$ crossing phantom model. QBI data sets with four $|q|$ values, $277,320,358$, and $392 \mathrm{~cm}^{-1}\left(b=3.0,4.0,5.0\right.$, and $6.0 \times 10^{3} \mathrm{~s} /$ $\mathrm{mm}^{2}$ ), were studied according to the results of the FWHM-nODF of the unidirectional capillaries, where the threshold to differentiate fibers crossing at $45^{\circ}$ was $|q|=320 \mathrm{~cm}^{-1}\left(b=4000 \mathrm{~s} / \mathrm{mm}^{2}\right)$. Each QBI data set was acquired with 162 diffusion-encoding directions (4-fold tessellated icosahedron), yielding an angular sampling resolution of $16^{\circ}$. By comparing QBI with the T2WI, the deviation angles, defined as the difference between the resolved separation angle and the reference separation angle (i.e. $45^{\circ}$ ), were measured in each voxel at the region containing the crossing capillaries.

\section{Angular resolution of $Q B I$ in a human brain}

A healthy human subject was recruited in order to quantify the angular resolution of QBI in a human brain. The subject provided an informed written consent following the guidelines of the Taipei Veteran General Hospital (TPE-VGH) internal review board. The corpus callosum, which mostly consists of compact fibers, served as the standard for the measurements. The FWHM-nODF of the human subject was measured using the same method employed with the phantom model. Sixty diffusion-encoding directions were uniformly encoded on a $\mathrm{q}$ circle, yielding an angular sampling resolution of $6^{\circ}$. Three coronal brain slices were imaged. The same $b$ values as used in the study of the unidirectional phantom model were applied, yielding $|q|$ values of $411,496,588,679$, and $753 \mathrm{~cm}^{-1}$ respectively. The total scan time was approximately $40 \mathrm{~min}$ with a number of excitation (NEX) of four. Additionally, QBI of the same brain slices was acquired with $|q|$ values of 411,588 , and $753 \mathrm{~cm}^{-1}\left(b=1.5,4.0,8.0 \times 10^{3} \mathrm{~s} / \mathrm{mm}^{2}\right)$. Each QBI data set was acquired with 162 diffusion-encoding directions and a NEX of three. The images were obtained at TPE-VGH using a 1.5 Tesla clinical MR scanner (Excite II; GE Medical Systems, Milwau-
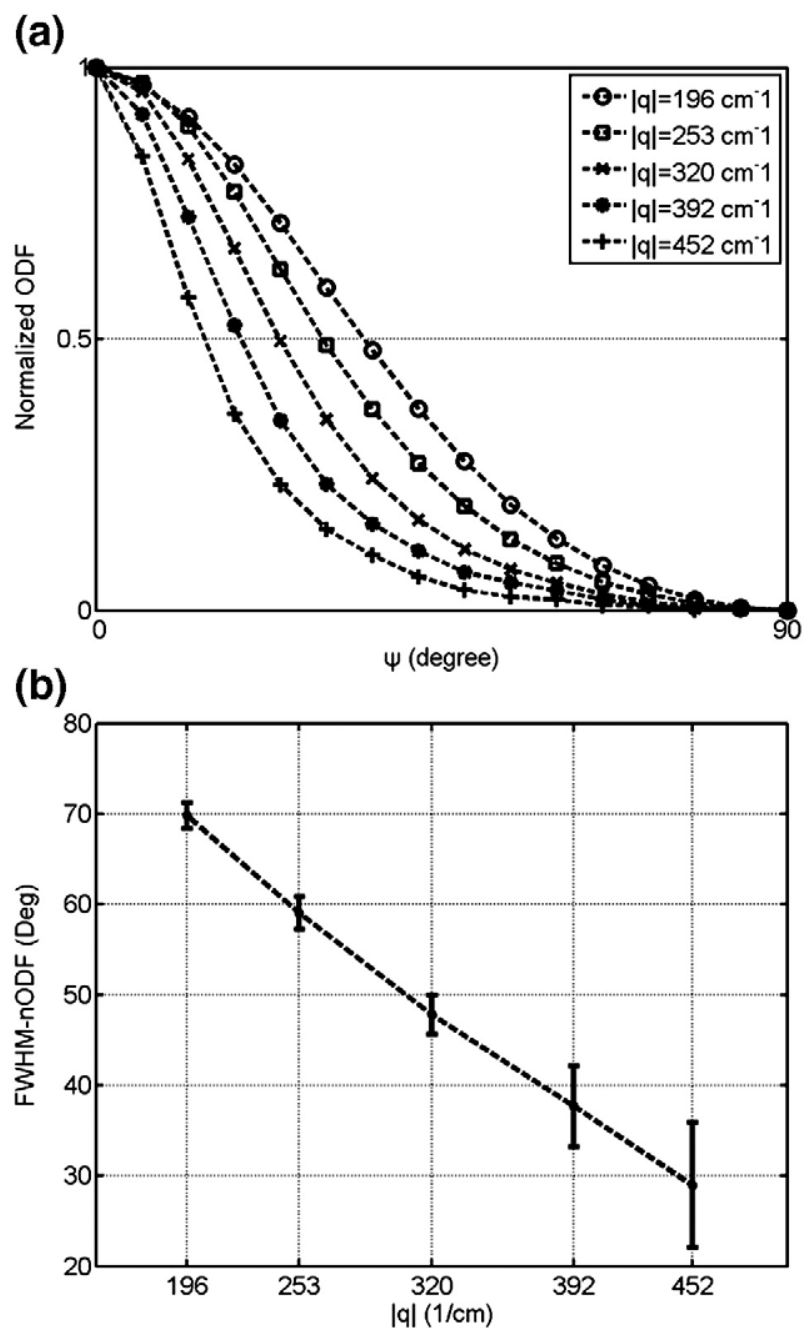

Fig. 4. (a) The distribution of the normalized ODF measured on the unidirectional phantom with various $|q|$ values. The $x$-axis showed the colatitudes, where $0^{\circ}$ and $90^{\circ}$ indicated the direction parallel to and perpendicular to the orientation of unidirectional phantom. (b) The result of FWHM-nODF measured in (a). 
kee, Wis., USA) equipped with an 8 channel head coil. DWIs were acquired using a single-shot diffusion spin-echo echo planar imaging (EPI) sequence with $\mathrm{TR} / \mathrm{TE}=3000 / 131.5 \mathrm{~ms}$, slice thickness $=3.6 \mathrm{~mm}, \mathrm{FOV}=23 \times 23 \mathrm{~cm}$ and matrix size $=64^{2}$, yielding an imaging resolution of $3.6 \mathrm{~mm}$. The total scan time was approximately $50 \mathrm{~min}$.

\section{QBI reconstruction}

QBI analysis was based on the reconstruction proposed by Tuch (Tuch, 2004),

$$
\mathrm{O}(u)=\int_{q \in u^{\perp}} \mathrm{E}(q) d q=\int \mathrm{E}(q) \delta\left(q^{T} u\right) d q,
$$

where $\mathrm{u}$ is the diffusion direction of interest, $\mathrm{E}(q)$ is the diffusion signal, and the $\mathrm{O}(u)$ is the ODF. During ODF reconstruction, the QBI data was interpolated using the sRBF (spherical Radial Basis Function) method to minimize the angular error caused by discrete sampling. A spherical Gaussian function with a width of $10^{\circ}$ was selected as the sRBF interpolation kernel by the condition number criterion (Tuch, 2004). The ODF was reconstructed with 2562 points, yielding an angular sampling resolution of $4^{\circ}$. The primary orientations of the ODF were then determined by selecting the greatest local $\mathrm{O}(u)$ in $3 \mathrm{D}$ space.

\section{Results}

Accuracy and angular resolution of QBI on phantom models

For each voxel, the angular error was calculated to assess the accuracy of QBI. The angular error was defined as the angle between the orientation estimated by QBI and the orientation of the phantom model. Then the mean and standard deviation (SD) of the angular error from all voxels were calculated and plotted as a function of $|q|$ value (Fig. 3). The SD of the angular error increased as the $|q|$ value increased. This was expected due to a lower SNR accompanied with severe signal attenuation. QBI with $|q|$ values of $196 \sim 320 \mathrm{~cm}^{-1}$ ( $b$ values of $1500 \sim 4000 \mathrm{~s} / \mathrm{mm}^{2}$ ) accurately resolved the orientation of compact capillaries. With $|q|$ values of 392 and $452 \mathrm{~cm}^{-1}$ $\left(b=6000\right.$ and $8000 \mathrm{~s} / \mathrm{mm}^{2}$ ), the orientation was accurately defined if an adequate SNR was reached. Despite the drawback of a lower SNR at higher $|q|$ values, a narrower FWHM-nODF (Fig. 4a) and consequently, an enhanced angular resolution (Fig. 4b) were achieved. The FWHM-nODF was $69.69^{\circ} \pm 1.43^{\circ}$ at $|q|=196 \mathrm{~cm}^{-1}$ $\left(b=1500 \mathrm{~s} / \mathrm{mm}^{2}\right)$ and decreased to $28.99^{\circ} \pm 6.91$ at $|q|=452 \mathrm{~cm}^{-1}$ $\left(b=8000 \mathrm{~s} / \mathrm{mm}^{2}\right)$.

In studying the $45^{\circ}$ crossing phantom model, $|q|$ values of 277 , 320,358 , and $392 \mathrm{~cm}^{-1}\left(b=3.0,4.0,5.0\right.$, and $\left.6.0 \times 10^{3} \mathrm{~s} / \mathrm{mm}^{2}\right)$ were chosen based on the FWHM-nODF derived from the unidirectional phantom. Fig. 5 illustrates the ODF maps and the primary orientations in the voxels located at the center of the

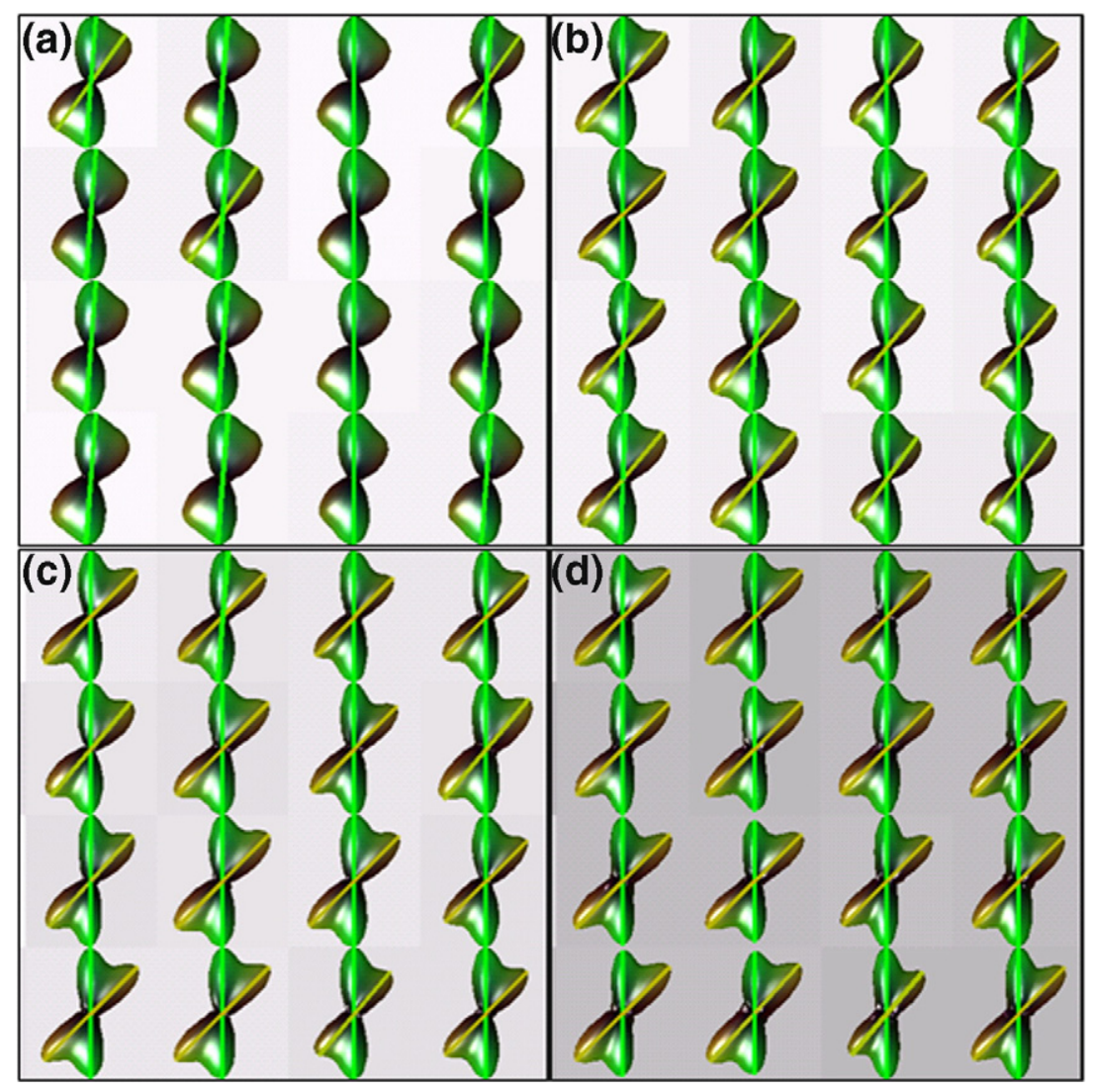

Fig. 5. The ODF maps and the primary orientations of QBI acquired on the crossing capillaries with the $45^{\circ}$ separation angle. The success rate (SR) and the accuracy of QBI increased when higher $|q|$ values were applied. The SR and the resolved separation angle were as follows, $\mathrm{SR}=27 \%, 32.50^{\circ} \pm 1.98^{\circ}$ at $|q|=$ $277 \mathrm{~cm}^{-1}\left(\mathrm{a}, b=3000 \mathrm{~s} / \mathrm{mm}^{2}\right), \mathrm{SR}=100 \%, 40.09^{\circ} \pm 2.72^{\circ}$ at $|q|=320 \mathrm{~cm}^{-1}\left(\mathrm{~b}, b=4000 \mathrm{~s} / \mathrm{mm}^{2}\right), \mathrm{SR}=100 \%, 43.63^{\circ} \pm 2.32^{\circ}$ at $|q|=358 \mathrm{~cm}^{-1}\left(\mathrm{c}, b=5000 \mathrm{~s} / \mathrm{mm}^{2}\right)$, and $\mathrm{SR}=100 \%, 44.31^{\circ} \pm 1.54^{\circ}$ at $|q|=392 \mathrm{~cm}^{-1}\left(\mathrm{~d}, b=6000 \mathrm{~s} / \mathrm{mm}^{2}\right)$. 
region containing crossing capillaries. The results show that crossing patterns could not be differentiated in some voxels with $q \mid=277 \mathrm{~cm}^{-1}$ ( $b=3000 \mathrm{~s} / \mathrm{mm}^{2}$, Fig. 5a). In this case, only $27 \%$ of the total voxels (33 voxels) were differentiated as crossing fibers and the separation angles were presented as $32.50^{\circ} \pm 1.98^{\circ}$ (mean \pm SD), which is far less than $45^{\circ}$. With $|q|=320,358$ and $392 \mathrm{~cm}^{-1}$ $\left(b=4000,5000\right.$ and $\left.6000 \mathrm{~s} / \mathrm{mm}^{2}\right)$, capillary crossings could be identified in all the voxels and the separation angles were $40.09^{\circ} \pm 2.72^{\circ}, 43.63^{\circ} \pm 2.32^{\circ}$ and $44.31^{\circ} \pm 1.54^{\circ}$, respectively. (Figs. $5 b$ to d).

\section{Angular resolution of $Q B I$ on a healthy subject}

In the human study, the FWHM-nODF was measured on the corpus callosum at five $|q|$ values (Fig. 6). The FWHM-nODF was $70.8^{\circ} \pm 5.2^{\circ}$ at $|q|=411 \mathrm{~cm}^{-1}\left(b=1500 \mathrm{~s} / \mathrm{mm}^{2}\right)$ and decreased to $50.7^{\circ} \pm 6.2^{\circ}$ at $|q|=753 \mathrm{~cm}^{-1}\left(b=8000 \mathrm{~s} / \mathrm{mm}^{2}\right)$. Fig. 7 shows the ODF maps of QBI with $|q|=411 \mathrm{~cm}^{-1}\left(b=1500 \mathrm{~s} / \mathrm{mm}^{2}\right.$, Fig. 7a), $588 \mathrm{~cm}^{-1}\left(b=4000 \mathrm{~s} / \mathrm{mm}^{2}\right.$, Fig. $\left.7 \mathrm{~b}\right)$ and $753 \mathrm{~cm}^{-1}\left(b=8000 \mathrm{~s} / \mathrm{mm}^{2}\right.$, Fig. 7c). In the region of the corpus callosum, QBI with various $|q|$ values resolved the unidirectional fiber structure within an individual voxel. The centrum semiovale, which contains three crossing fibers [corona radiata (CR), superior longitudinal fasciculus (SLF), and the striations of the corpus callosum (CC)], was studied to assess the angular resolution with respect to $|q|$ values. Fig. $7 \mathrm{~d}$ shows the ODF of six voxels at three $|q|$ values. Voxels 1 and 2 show the three-way crossing fibers of the CC, CR, and SLF. QBI could clearly differentiate the three fibers at a $|q|$ value of $753 \mathrm{~cm}^{-1} \quad\left(b=8000 \mathrm{~s} / \mathrm{mm}^{2}\right)$. QBI with $|q|=588 \mathrm{~cm}^{-1}$ $\left(b=4000 \mathrm{~s} / \mathrm{mm}^{2}\right)$ resolved both the CC and CR, while the SLF was obscure. With $|q|=411 \mathrm{~cm}^{-1}\left(b=1500 \mathrm{~s} / \mathrm{mm}^{2}\right)$, QBI resolved only one or two distinct fiber directions. Voxels 3 and 4 show the two-way crossing fibers of the $\mathrm{CC}$ and $\mathrm{CR}$, which could be distinguished by QBI at $|q|=588$ and $753 \mathrm{~cm}^{-1}(b=4000$ and $\left.8000 \mathrm{~s} / \mathrm{mm}^{2}\right)$. With $|q|=411 \mathrm{~cm}^{-1}\left(b=1500 \mathrm{~s} / \mathrm{mm}^{2}\right)$, the crossing fibers could not be detected by QBI. Voxels 5 and 6 show that the $\mathrm{CC}$ and CR could only be resolved by QBI at a $|q|$ value of $753 \mathrm{~cm}^{-1}\left(b=8000 \mathrm{~s} / \mathrm{mm}^{2}\right)$.

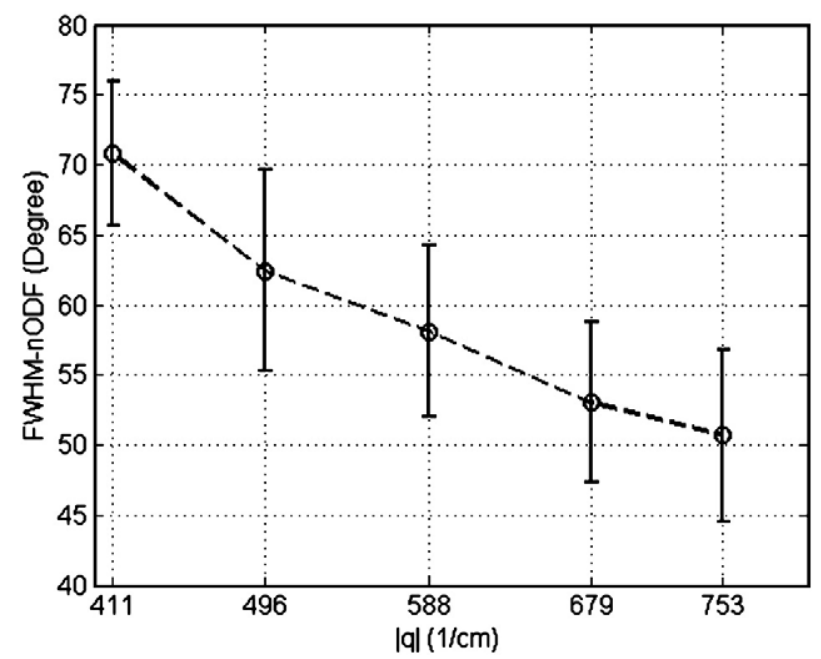

Fig. 6. The results of the FWHM-nODF, measured on the corpus callosum of a healthy subject, plotted as a function of $|q|$ value. A narrower FWHMnODF was achieved as the $|q|$ value increased.

\section{Discussion}

By combining QBI with the T2WI of the phantom models, we quantified the accuracy and angular resolution of QBI in the resolution of fiber crossings at various $|q|$ values. The results show that QBI with either a low $|q|$ or a high $|q|$ value can accurately detect the orientation of compact capillaries if an adequate imaging SNR can be achieved. At high $|q|$ values, the angular resolution could be improved despite the reciprocal decrease of the SNR. On the contrary, QBI with low $|q|$ values could not resolve fibers with small separation angles and resulted in the vague estimation of fiber orientations. By estimating the FWHM-nODF on the corpus callosum of a healthy subject, QBI with $|q|=411$ and $753 \mathrm{~cm}^{-1}$ $\left(b=1500\right.$ and $8000 \mathrm{~s} / \mathrm{mm}^{2}$ ) achieved an angular resolution of $70.8^{\circ}$ and $50.7^{\circ}$, respectively. It was demonstrated on both the phantom model and the human brain that QBI encoding with low $|q|$ values may result in underestimation of the angle between the crossing fibers.

\section{FWHM-nODF}

The ability of QBI to resolve intersecting capillaries can be quantified by adopting the FWHM-nODF which is obtained by applying a simplified scheme as used in this study. As shown in Fig. 4a, QBI with high $|q|$ values leads to a small FWHM-nODF and improved angular resolution. This can be explained with a defined spatial resolution $\left(\Delta r=\alpha_{0} / 2 \pi q\right)$ in which a high $|q|$ value improves the resolution (Tuch, 2004). Although a better angular resolution is achieved by QBI with a high $|q|$ value, the lower SNR results in considerable uncertainty.

\section{Accuracy of $Q B I$}

Compact and intersecting capillaries were used to quantify the accuracy of QBI to define the fiber orientation. Water-filled capillaries with an i.d. of $20 \mu \mathrm{m}$ and an o.d. of $90 \mu \mathrm{m}$ afforded a restrictive environment to simulate the crossing of axonal fibers in a biological situation. Similar phantoms have been used to simulate the displacement distribution of the diffusion tensor (von dem Hagen and Henkelman, 2002) and to validate the accuracy of DSI to define fiber crossings (Lin et al., 2003). Thus, it is legitimate to assess the accuracy of QBI in the crossing capillaries with these models.

For the compact capillaries, our results show that the accuracy of QBI to estimate the single fiber orientation depended on the $|q|$ value and the available imaging SNR. QBI using a higher $|q|$ value was generally accompanied with severe diffusion signal decay and T2 attenuation. With adequate imaging SNR, the accuracy of QBI to define the single fiber orientation was well preserved at various $|q|$ values (Fig. 3). For this capillary phantom, optimum $|q|$ values for QBI to define the single fiber orientation ranged between $|q|=196$ and $320 \mathrm{~cm}^{-1}\left(b=1500\right.$ and $\left.4000 \mathrm{~s} / \mathrm{mm}^{2}\right)$ and the SNR of the reference image was 11 (for the case of one average). This is not controversial since a high $|q|$ value decreases the imaging SNR and consequently dilutes the accuracy.

According to the FWHM-nODF measurement on compact fibers, QBI with $|q|=277 \mathrm{~cm}^{-1}\left(b=3000 \mathrm{~s} / \mathrm{mm}^{2}\right)$ was inadequate to define crossing in the $45^{\circ}$ crossing phantom model. It resulted in only identifying $27 \%$ of the total voxels as having a crossing structure and the measured separation angle was far less than the phantom design. This result implies that QBI with a low $|q|$ value 


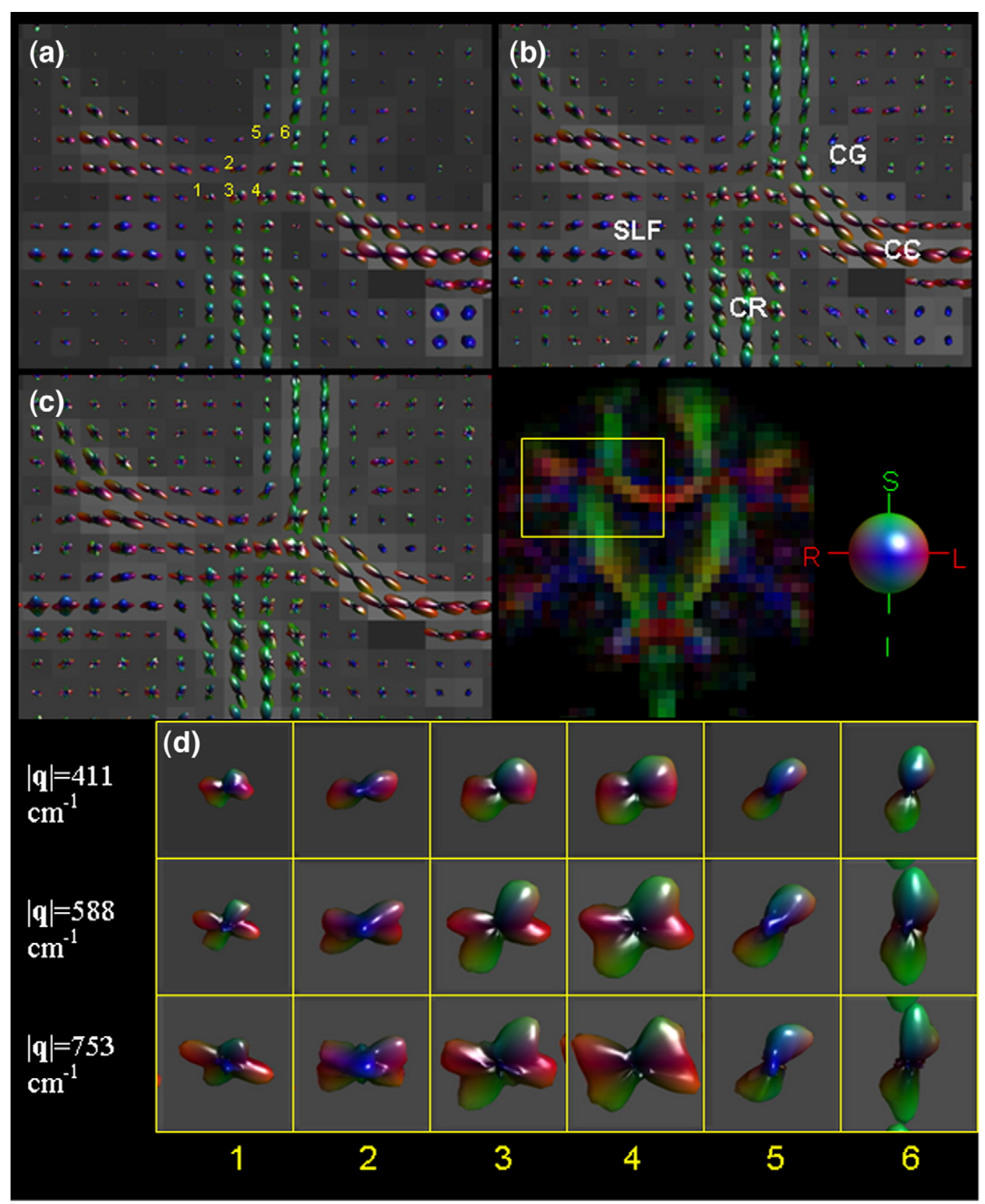

Fig. 7. The QBI on the coronal brain slice of a normal subject. The $|q|$ value was $411\left(\mathrm{a}, b=1500 \mathrm{~s} / \mathrm{mm}^{2}\right), 588\left(\mathrm{~b}, b=4000 \mathrm{~s} / \mathrm{mm}^{2}\right)$, and $753\left(\mathrm{c}, b=8000 \mathrm{~s} / \mathrm{mm}^{2}\right)$ $\mathrm{cm}^{-1}$ respectively. The RGB image of the coronal brain slice was color-coded according to (red, green, blue) $=\mathrm{FA} \times|e|$, where $e$ was the principle eigenvector of the DTI. The yellow ROI was the crossing region of the corona radiata (CR), corpus callosum (CC), and superior longitudinal fasciculus (SLF). Six voxels were selected to compare the ODFs across various $|q|$ values (d). The $|q|$ values of the top, middle and bottom row were $411,588,753 \mathrm{~cm}^{-1}(b=1500,4000$, and $8000 \mathrm{~s} / \mathrm{mm}^{2}$ ) respectively.

may lead to an underestimation of the separation angle. The same condition also occurred for QBI with a $|q|$ value of $320 \mathrm{~cm}^{-1}$ $\left(b=4000 \mathrm{~s} / \mathrm{mm}^{2}\right)$. Despite the fact that fiber crossings were identified in all the voxels, the estimated separation angle was underestimated and the deviation angle was $-4.91^{\circ} \pm 2.72^{\circ}$. With $|q|=358$ and $392 \mathrm{~cm}^{-1}\left(b=5000\right.$ and $\left.6000 \mathrm{~s} / \mathrm{mm}^{2}\right)$, all crossing patterns were identified and the errors were $-1.37^{\circ} \pm 2.32^{\circ}$ and $-0.69^{\circ} \pm 1.54^{\circ}$ respectively. The result was satisfactory and the error was close to the noise level. The deviation may have been comprised of the error related to MR noise and a bias inherent to the HARDI discrete sampling and ODF reconstruction.

\section{QBI parameters for human study}

The FWHM-nODF of the CC was adopted as a standard to estimate the angular resolution of QBI in relation to various $|q|$ values. The angular resolution of QBI was enhanced to $50.7^{\circ}$ as the 
$|q|$ value increased to $753 \mathrm{~cm}^{-1}$. Despite deterioration of the angular resolution in comparison with the capillary models, the trend where higher $|q|$ values yield a better angular resolution was similar to that of the phantom models (Figs. $3 \mathrm{~b}$ and 6). The difference of the angular resolution between the phantom models and the human tracts may be due to the different physical characteristics between human brain tracts and the phantom capillaries. These characteristics may include properties such as permeability, diffusivity, fiber diameter, and etc. In addition, a short diffusion time in the clinical scanner was required to minimize the echo time, which made the measured ODF smooth (Assaf et al., 2000) and consequently diluted the sensitivity of QBI to define the molecular displacement.

In the brain region of the centrum semiovale, three elements (CR, CC, and SLF) are intersected. QBI with a low $|q|$ value revealed less anatomical information compared to QBI with a high $|q|$ value (Fig. 7). This underestimation was consistent with the results of the phantom models. Thus, it is possible that QBI encoding with an inadequate $|q|$ value fails to completely resolve architectural information. Tuch et al. showed that high-frequency QBI $\left(|q|=950 \mathrm{~cm}^{-1}\right.$, $b=12,000 \mathrm{~s} / \mathrm{mm}^{2}$ ) revealed additional anatomical structure in comparison to low-frequency QBI $\left(|q|=670 \mathrm{~cm}^{-1}, b=4000 \mathrm{~s} / \mathrm{mm}^{2}\right)$. At the brain area between the $\mathrm{CR}$ and the striation of the $\mathrm{CC}$, lowfrequency $\mathrm{QBI}$ was less consistent than high-frequency $\mathrm{QBI}$ in showing fiber connections at the intersecting area along the $\mathrm{CR}$ and the CC (Tuch et al., 2003). This underestimation supports our inference and should be avoided for accurate tractography.

Alexander and Barker found optimal $b$ values for two-fiber measurements using the Levenberg-Marquardt algorithm and Monte-Carlo simulation. Taking the clinical scanner limitations and the optimal TE into consideration, optimum $b$ values for twofiber measurements were concluded to be 2200 and $2800 \mathrm{~s} / \mathrm{mm}^{2}$ for typical brain-tissue diffusivity. Although the fractional anisotropy and the mean diffusivity were well estimated, the ability to resolve separation angle was not clarified (Alexander and Barker, 2005). We were only able to conclude a rule that low-frequency QBI, such as $|q|=196 \mathrm{~cm}^{-1}\left(b=1500 \mathrm{~s} / \mathrm{mm}^{2}\right)$, can be used to map crossing fiber bundles with a separation angle of $70.8^{\circ}$, while highfrequency QBI, such as $|q|=452 \mathrm{~cm}^{-1}\left(b=8000 \mathrm{~s} / \mathrm{mm}^{2}\right)$, can be applied to resolve fibers with a separation angle of $50.7^{\circ}$ if an adequate SNR can be achieved.

\section{Factors that might affect the accuracy of $Q B I$}

The accuracy of QBI in resolving fiber orientations may be affected by both the imaging parameters and the post-processing method. During image acquisition, the angular resolution of QBI to define crossing fibers can be improved by higher $|q|$ values despite the fact that the SNR reciprocally decreases due to severe signal attenuation. This is because the defined spatial resolution is enhanced (Tuch, 2004) and the FWHM-nODF is narrowed (Fig. 4a). At the same $b$ value, a long diffusion time can increase the measurement of tissue anisotropy and reduce uncertainty regarding the estimated direction of fibers. However, this would result in a long echo time and again decrease the SNR (Assaf et al., 2000; Kim et al., 2005). Meanwhile, a long diffusion time yielding a small $|q|$ value under the same $b$ value results in the low-pass effect by the Bessel beam and makes the ODF of QBI smoother than the true ODF, consequently reducing the angular resolution (Tuch, 2004). There is obviously a tradeoff between diffusion time and $|q|$ value for QBI encoding at the same $b$ value when using FWHM-nODF. Alexander and Barker suggested that a shorter TE may yield a better SNR for the selected $b$ value on a clinical scanner. In our experience, the diffusion time with an Einstein length of molecular diffusion close to the diameter of the axonal fiber was suggested (Li et al., 2006; Lin et al., 2003). In this way, an optimum $|q|$ value can be defined. Furthermore, an infinitesimal diffusion gradient pulse width is generally suggested for the $q$ space imaging method (Callaghan, 1993). As the duration of gradient increases, the local maxima and minima of $\mathrm{E}(q)$ will shift to the right on the qa axis (Blees, 1994), indicating an underestimation of the microstructural dimensions, $a$. This underestimation may cause problems in determining the actual dimensions of the microstructure but it may not affect the accuracy of QBI to define fiber orientations if the separation angle is larger than the limited angular resolution. Assaf et al. suggested that the finite gradient pulse width can make the PDF narrower and more intense than that under the narrow pulse approximation (Assaf et al., 2002). This may consequently increase the ability to resolve separate fibers. Furthermore, the quantity of diffusion-encoding directions has been shown as a significant factor for uncertainty in DTI (Jones, 2004). In HARDI, more diffusion-encoding directions, although prolonging the acquisition time, may enhance the accuracy and the sampling resolution of QBI.

At the image post-processing stage, selection of the interpolation kernel and the smooth kernel may influence the accuracy of QBI as well (Tuch, 2004). In this study, the condition number was calculated to optimize the interpolated kernel width but the smooth kernel was not applied due to its reciprocal influence between accuracy and the angular resolution. Finally, the ODF reconstruction method may have an effect on the angular resolution. Reconstruction using spherical harmonics with a high model order may increase the angular resolution and minimize the estimated error from insufficient angular discrimination at low $b$ value encoding (Hess et al., 2006).

\section{Conclusion}

Using capillary phantoms, we quantified the accuracy and the angular resolution of QBI to define fiber orientations. With both low and high $|q|$ values, QBI could define compact fiber orientations with an error of less than $2.5^{\circ}$. In resolving crossing fibers, a high $|q|$ value facilitated the ability of QBI to define the separation angle if an adequate SNR could be achieved. The angular resolution could be estimated by measuring the FWHMnODF. On the contrary, low $|q|$ values yield a high SNR but may have resulted in an underestimation of small intersecting angles. In conclusion, the ability of QBI to resolve crossing fibers in a voxel is highly dependent on the selected $|q|$ value. This result suggests that future tractography algorithms can be designed to account for the underestimation error based on the encoding $|q|$ value.

\section{Acknowledgments}

This study is supported in part by the National Science Council Grant 96-2752-H-010-004-PAE, and the authors thank Dave Tuch and Chen Chang for their help on the study. The authors also acknowledge technical support from the Functional and MicroMagnetic Resonance Imaging Center supported by the National Research Program for Genomic Medicine, National Science Council, Taiwan, ROC (NSC96-3112-B-001-009). JDT is grateful to the National Health and Medical Research Council (NHMRC) of Australia and Austin Health for support. 


\section{Appendix}

An encoding scheme was developed to measure the FWHMnODF of compact fibers within a reasonable time period. We posited that the uniform fibers within each MR voxel were axially symmetrical and therefore both the diffusion signal $\mathrm{E}(q)$ and the $\mathrm{ODF} \mathrm{O}(u)$ were axially symmetric functions. In this way, the encoding scheme could be simplified to a circle from a sphere in $q$ space. To define the encoding plane, diffusion tensor imaging was applied. The encoding circle was defined by the plane containing the first eigenvector of the diffusion tensor. The FWHM-nODF was measured using the following derived equation. To simplify the explanation, the fiber was assumed to lie on the $z$-axis without losing generality (Fig. 2c). Using spherical coordinates, the vectors $u$ and $q$ were represented as $\left(r_{u}, \theta_{u}\right.$, $\left.\psi_{u}\right)$ and $\left(r_{q}, \theta_{q}, \psi_{q}\right)$ respectively, where $\theta_{u}$ and $\theta_{q}$ were longitudes and $\psi_{u}$ and $\psi_{q}$ were colatitudes. Eq. (1) was therefore rewritten as

$$
\begin{aligned}
\mathrm{O}\left(r_{u}, \theta_{u}, \psi_{u}\right)= & \int \mathrm{E}\left(r_{q}, \theta_{q}, \psi_{q}\right) \delta\left(r _ { q } r _ { u } \left(\cos \theta_{q} \sin \psi_{q} \cos \theta_{u} \sin \psi_{u}\right.\right. \\
& +\sin \theta_{q} \sin \psi_{q} \sin \theta_{u} \sin \psi_{u} \\
& \left.\left.+\cos \psi_{q} \cos \psi_{u}\right)\right) r_{q} d r_{q} d \theta_{q} d \psi_{q}
\end{aligned}
$$

According to the assumption that $\mathrm{E}(q)$ and $\mathrm{O}(u)$ were axially symmetrical functions, $\mathrm{E}\left(r_{q}, \theta_{q}, \psi_{q}\right)$ and $\mathrm{O}\left(r_{u}, \theta_{u}, \psi_{u}\right)$ was described as

$\mathrm{E}\left(r_{q}, \theta_{q}, \psi_{q}\right)=\mathrm{E}\left(r_{q}, 0, \psi_{q}\right)$ for all $\theta_{q}$

$\mathrm{O}\left(r_{u}, \theta_{u}, \psi_{u}\right)=\mathrm{O}\left(r_{u}, 0, \psi_{u}\right)$ for all $\theta_{u}$

At wave vector $|q|=q^{\prime} \mathrm{O}\left(r_{u}, 0, \psi_{u}\right)$ can be written as

$$
\begin{aligned}
& \mathrm{O}\left(r_{u}, 0, \psi_{u}\right) \\
& \quad=\int \mathrm{E}\left(r_{q}, 0, \psi_{q}\right) \delta\left(\cos \theta_{q} \sin \psi_{q} \sin \psi_{u}+\cos \psi_{q} \cos \psi_{u}\right) d \theta_{q} \mathrm{~d} \psi_{q} \\
& \quad=q^{\prime} \int \mathrm{E}\left(q, 0, \psi_{q}\right) \delta\left(\psi_{q}-\tan ^{-1}\left(-\cot \psi_{u} \sec \theta_{q}\right)\right) d \theta_{q} d \psi_{q}
\end{aligned}
$$

$\mathrm{O}\left(r_{u}, 0, \psi_{u}\right)$ can be simplified by considering the integral over the plane $\mathrm{d} \psi_{q}$,

$\mathrm{O}\left(r_{u}, 0, \psi_{u}\right)=q^{\prime} \int \mathrm{E}\left(q, 0, \tan ^{-1}\left(-\cot \psi_{u} \sec \theta_{q}\right)\right) d \theta_{q}$

Fig. 2b showed an illustration of the $\mathrm{E}(q)$ measured as a function of the colatitudes $\psi_{q}$, which also represented the angle between the fiber axis and the diffusion gradient direction. With a given $\mathrm{O}\left(r_{u}, 0\right.$, $\psi_{u}$ ), the diffusion signals at the points of the corresponding equator, shown in Fig. 2c, could be easily obtained according to Eq. (6). For example, the diffusion signals at points $\mathrm{A}, \mathrm{B}$, and $\mathrm{C}$ shown in Fig. 2c correspond to the signals at points $\mathrm{A}, \mathrm{B}$, and $\mathrm{C}$ shown in Fig. 2b, respectively. By varying $\psi_{u}$ from 0 to $\pi$, the ODF of uniform fibers is measured using the simplified encoding scheme. The ODF is then min-max normalized, i.e. linearly mapping the values to the range between 0 and 1 . The FWHM was measured on the normalized ODF to evaluate the angular resolution of QBI (Fig. 2d).

\section{References}

Alexander, D.C., Barker, G.J., 2005. Optimal imaging parameters for fiberorientation estimation in diffusion MRI. NeuroImage 27, 357-367.
Alexander, A.L., Hasan, K.M., Lazar, M., Tsuruda, J.S., Parker, D.L., 2001. Analysis of partial volume effects in diffusion-tensor MRI. Magn. Reson. Med. 45, 770-780.

Alexander, D.C., Barker, G.J., Arridge, S.R., 2002. Detection and modeling of non-Gaussian apparent diffusion coefficient profiles in human brain data. Magn. Reson. Med. 48, 331-340.

Anderson, A.W., 2005. Measurement of fiber orientation distributions using high angular resolution diffusion imaging. Magn. Reson. Med. 54, 1194-1206.

Assaf, Y., Cohen, Y., 1999. Structural information in neuronal tissue as revealed by q-space diffusion NMR spectroscopy of metabolites in bovine optic nerve. NMR Biomed. 12, 335-344.

Assaf, Y., Basser, P.J., 2005. Composite hindered and restricted model of diffusion (CHARMED) MR imaging of the human brain. NeuroImage $27,48-58$.

Assaf, Y., Mayk, A., Cohen, Y., 2000. Displacement imaging of spinal cord using q-space diffusion-weighted MRI. Magn. Reson. Med. 44, 713-722.

Assaf, Y., Ben-Bashat, D., Chapman, J., Peled, S., Biton, I.E., Kafri, M., Segev, Y., Hendler, T., Korczyn, A.D., Graif, M., Cohen, Y., 2002. High b-value q-space analyzed diffusion-weighted MRI: application to multiple sclerosis. Magn. Reson. Med. 47, 115-126.

Basser, P.J., Mattiello, J., LeBihan, D., 1994. MR diffusion tensor spectroscopy and imaging. Biophys. J. 66, 259-267.

Basser, P.J., Pajevic, S., Pierpaoli, C., Duda, J., Aldroubi, A., 2000. In vivo fiber tractography using DT-MRI data. Magn. Reson. Med. 44, $625-632$.

Behrens, T.E., Johansen-Berg, H., Woolrich, M.W., Smith, S.M., WheelerKingshott, C.A., Boulby, P.A., Barker, G.J., Sillery, E.L., Sheehan, K., Ciccarelli, O., Thompson, A.J., Brady, J.M., Matthews, P.M., 2003. Non-invasive mapping of connections between human thalamus and cortex using diffusion imaging. Nat. Neurosci. 6, 750-757.

Blees, M.H., 1994. The effect of finite duration of gradient pulses on the pulsed-field-gradient NMR method for studying restricted diffusion. J. Magn. Reson. Ser. A 109, 203-209.

Callaghan, P.T., 1993. Principles of Nuclear Magnetic Resonance Microscopy.

Campbell, J.S., Siddiqi, K., Rymar, V.V., Sadikot, A.F., Pike, G.B., 2005. Flow-based fiber tracking with diffusion tensor and q-ball data: validation and comparison to principal diffusion direction techniques. NeuroImage 27, 725-736.

Conturo, T.E., Lori, N.F., Cull, T.S., Akbudak, E., Snyder, A.Z., Shimony, J.S., McKinstry, R.C., Burton, H., Raichle, M.E., 1999. Tracking neuronal fiber pathways in the living human brain. Proc. Natl. Acad. Sci. U. S. A. 96, 10422-10427.

Descoteaux, M., Angelino, E., Fitzgibbons, S., Deriche, R., 2006. Apparent diffusion coefficients from high angular resolution diffusion imaging: estimation and applications. Magn. Reson. Med. 56 (2), 395-410.

Frank, L.R., 2001. Anisotropy in high angular resolution diffusion-weighted MRI. Magn. Reson. Med. 45, 935-939.

Frank, L.R., 2002. Characterization of anisotropy in high angular resolution diffusion-weighted MRI. Magn. Reson. Med. 47, 1083-1099.

Grannell, P.K., Mansfield, P., 1975. Microscopy in vivo by nuclear magnetic resonance. Phys. Med. Biol. 20, 477-482.

Hess, C.P., Mukherjee, P., Han, E.T., Xu, D., Vigneron, D.B., 2006. Q-ball reconstruction of multimodal fiber orientations using the spherical harmonic basis. Magn. Reson. Med. 56, 104-117.

Jones, D.K., 2004. The effect of gradient sampling schemes on measures derived from diffusion tensor MRI: a Monte Carlo study. Magn. Reson. Med. 51, 807-815.

Kim, S., Chi-Fishman, G., Barnett, A.S., Pierpaoli, C., 2005. Dependence on diffusion time of apparent diffusion tensor of ex vivo calf tongue and heart. Magn. Reson. Med. 54, 1387-1396.

King, M.D., Houseman, J., Roussel, S.A., van Bruggen, N., Williams, S.R., Gadian, D.G., 1994. q-Space imaging of the brain. Magn. Reson. Med. 32, 707-713.

Li, Y.C., Cho, K.H., Chou, K.H., Lin, C.P., 2006. Optimal imaging parameters for minimum angular discrimination in diffusion spectrum imaging. Proc ISMRM 14th Ann Meeting, Seattle, USA, 644. 
Lin, C.P., Tseng, W.Y., Cheng, H.C., Chen, J.H., 2001. Validation of diffusion tensor magnetic resonance axonal fiber imaging with registered manganese-enhanced optic tracts. NeuroImage 14, 1035-1047.

Lin, C.P., Wedeen, V.J., Chen, J.H., Yao, C., Tseng, W.Y., 2003. Validation of diffusion spectrum magnetic resonance imaging with manganeseenhanced rat optic tracts and ex vivo phantoms. NeuroImage 19, 482-495.

Mori, S., Crain, B.J., Chacko, V.P., van Zijl, P.C., 1999. Three-dimensional tracking of axonal projections in the brain by magnetic resonance imaging. Ann. Neurol. 45, 265-269.

Ozarslan, E., Mareci, T.H., 2003. Generalized diffusion tensor imaging and analytical relationships between diffusion tensor imaging and high angular resolution diffusion imaging. Magn. Reson. Med. 50, 955-965.

Perrin, M., Poupon, C., Cointepas, Y., Rieul, B., Golestani, N., Pallier, C., Riviere, D., Constantinesco, A., Le Bihan, D., Mangin, J.F., 2005a. Fiber tracking in q-ball fields using regularized particle trajectories. Inf. Process Med. Imag. 19, 52-63.

Perrin, M., Poupon, C., Rieul, B., Leroux, P., Constantinesco, A., Mangin, J.F., Lebihan, D., 2005b. Validation of q-ball imaging with a diffusion fibre- crossing phantom on a clinical scanner. Philos. Trans. R. Soc. Lond., B Biol. Sci. 360, 881-891.

Tournier, J.D., Calamante, F., Gadian, D.G., Connelly, A., 2004. Direct estimation of the fiber orientation density function from diffusion-weighted MRI data using spherical deconvolution. NeuroImage 23, 1176-1185.

Tuch, D.S., 2004. Q-ball imaging. Magn. Reson. Med. 52, 1358-1372.

Tuch, D.S., Reese, T.G., Wiegell, M.R., Makris, N., Belliveau, J.W., Wedeen, V.J., 2002. High angular resolution diffusion imaging reveals intravoxel white matter fiber heterogeneity. Magn. Reson. Med. 48, 577-582.

Tuch, D.S., Reese, T.G., Wiegell, M.R., Wedeen, V.J., 2003. Diffusion MRI of complex neural architecture. Neuron 40, 885-895.

von dem Hagen, E.A., Henkelman, R.M., 2002. Orientational diffusion reflects fiber structure within a voxel. Magn. Reson. Med. 48, 454-459.

Wiegell, M.R., Larsson, H.B., Wedeen, V.J., 2000. Fiber crossing in human brain depicted with diffusion tensor MR imaging. Radiology 217, 897-903.

Zhan, W., Gu, H., Xu, S., Silbersweig, D.A., Stern, E., Yang, Y., 2003. Circular spectrum mapping for intravoxel fiber structures based on high angular resolution apparent diffusion coefficients. Magn. Reson. Med. 49, 1077-1088 